\title{
Well-definedness of Streams by Termination
}

\author{
Hans Zantema \\ Department of Computer Science, TU Eindhoven, P.O. Box 513, \\ 5600 MB Eindhoven, The Netherlands, H.Zantema@tue.nl \\ and \\ Institute for Computing and Information Sciences, Radboud University \\ Nijmegen, P.O. Box 9010, 6500 GL Nijmegen, The Netherlands
}

\begin{abstract}
Streams are infinite sequences over a given data type. A stream specification is a set of equations intended to define a stream. We propose a transformation from such a stream specification to a TRS in such a way that termination of the resulting TRS implies that the stream specification admits a unique solution. As a consequence, proving such well-definedness of several interesting stream specifications can be done fully automatically using present powerful tools for proving TRS termination.
\end{abstract}

\section{Introduction}

Streams are among the simplest data types in which the objects are infinite. We consider streams to be maps from the natural numbers to some data type $D$. The basic constructor for streams is the operator ':' mapping a data element $d$ and a stream $s$ to a new stream $d: s$ by putting $d$ in front of $s$. Using this operator we can define streams by equations. For instance, the stream zeros only consisting of 0's can be defined by the single equation zeros $=0$ : zeros. More complicated streams are defined using stream functions. For instance, the boolean Fibonacci stream $\mathrm{Fib}$ is defined ${ }^{1}$ to be the fixpoint of the function $f$ defined by

$$
f(0: \sigma)=0: 1: f(\sigma), \quad f(1: \sigma)=0: f(\sigma) .
$$

It turns out that Fib $=0: c$ for the stream $c$ defined by $c=1: f(c)$. Although these stream definitions are extremely simple, the resulting streams are typically non-periodic and have remarkable properties. For instance, one can make a turtle visualization (see also http://www.win.tue.nl/ ^hzantema/str.html) as follows. Choose an initial drawing direction and traverse the elements of the stream Fib as follows: if the symbol 0 is read then the drawing direction is moved 30 degrees to the right; if the symbol 1 is read then the drawing direction is moved 150 degrees to the left. In both cases after doing so a line of unit length is drawn. Then after 200.000 steps the following picture is obtained.

\footnotetext{
${ }^{1}$ In [1] it is called infinite Fibonacci word. It can also be defined as the limit of the strings $\phi_{i}$ where $\phi_{1}=1, \phi_{2}=0, \phi_{i+2}=\phi_{i+1} \phi_{i}$ for $i \geq 1$, showing the relationship with Fibonacci numbers.
} 


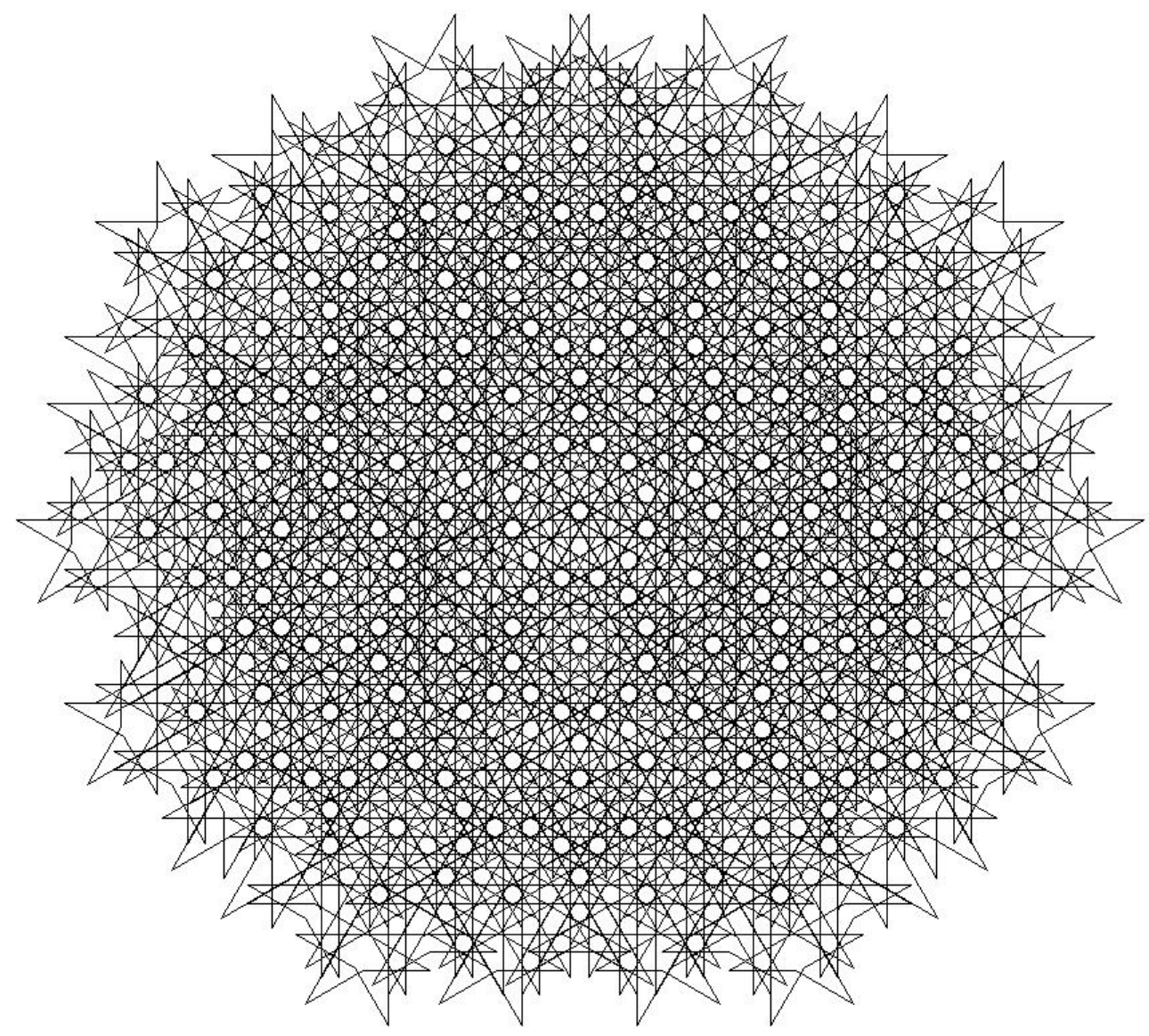

Streams have been studied extensively, e.g. in [1]. In this paper we consider stream specifications consisting of a set of equations like above we did for the Fibonacci stream. We address the most fundamental question one can think of: does such a set of equations admits a unique solution as constants and functions on streams? This is not always the case. For instance, every $f$ mapping $x: \sigma$ to $x: c$ for any constant stream $c$ satisfies the stream specification

$$
f(x: \sigma)=x: g(f(\sigma)), \quad g(x: \sigma)=\sigma,
$$

where $g$ is the tail function removing the first element of the stream.

Intuitively this notion of well-definedness is closely related to termination of the process of unfolding definitions. The past ten years showed up a remarkable progress in techniques and implementations for proving termination of rewrite systems $[2,5,9]$. One of the objectives of this paper is to exploit this power for proving well-definedness of stream specifications. In our approach we introduce fresh operators head and tail intended to observe streams. We present a transformation of the specification to its observational variant. This is a TRS mimicking 
the stream specification in such a way that head or tail applied on any stream constant or stream function can always be rewritten. This transformation is straightforward and easy to implement; an implementation for boolean stream specifications, both in Windows an Linux, together with several examples, is found in http://www.win.tue.nl/ ${ }^{\sim}$ hzantema/str.zip.

The main result of this paper states that if the observational variant of a specification is terminating, then the specification admits a unique solution. It turns out that for several interesting cases termination of the observational variant of a specification can be proved by termination tools like AProVE [4] or TTT2 [7]. This provides a new technique to prove well-definedness of stream specifications fully automatically, applying for cases where earlier approaches fail. Our main result appears in two variants:

- a variant restricting to ground terms for general stream specifications (Theorem 1), and

- a variant generalizing to all streams for stream specifications not depending on particular data elements (Theorem 2).

By an example we show that the approach does not work for general stream specifications and functions applied on all streams. Moreover, we show that our technique is not complete: the fixpoint definition of the Fibonacci stream as we just gave is a well-defined stream specification for which the observational variant is non-terminating.

Proving well-definedness in stream specification is closely related to proving equality of streams. A standard approach for this is co-induction [11]: two streams or stream functions are equal if a bisimulation can be found between them. Finding such an arbitrary bisimulation is a hard problem in the general setting, but restricting to circular co-induction [6] finding this automatically is tractable. A strong tool doing so is Circ [8]. The tool Circ focuses on proving equality, but proving well-definedness of a function $f$ can also be proved by equality as long as the equations for $f$ are orthogonal: take a copy $f^{\prime}$ of $f$ with the same equations, and prove $f=f^{\prime}$. For many examples this works well, but there are also small stream specifications for which our approach succeeds in proving well-definedness and Circ fails. Conversely our approach can be used to prove equality of two streams: if one stream satisfies the specification of the other one, and this specification is well-defined, then the streams are equal. The input format of Circ differs from what we call stream specifications: head and tail are already building blocks and the Circ input is essentially the same as what we call the observational variant.

Another closely related topic is productivity of stream specifications, as studied by [3]. Productive stream specifications are always well-defined. Conversely we will give an example (Example 4) of a stream specification that is well-defined, but not productive. Our format of stream specifications is strongly inspired by [3]. In [3] a technique is developed for establishing productivity fully automatically for a restricted class of stream specifications. In particular, only a very mild type of nesting in the right hand sides of the rule is allowed. Our technique typically applies where these restrictions do not hold. 
Both stream equality [10] and productivity [12] have been proved to be $\Pi_{2}^{0}$ complete, hence undecidable. By similar Turing machine construction the same is expected to hold for stream well-definedness.

This paper is structured as follows. In Section 2 we present the basics of stream specifications and their models. In Section 3 we define the transformation of a stream specification to its observational variant. In Section 4 we present and prove the main theorem: if the observational variant is terminating then restricted to ground terms the specification has a unique model. In Section 5 we show that this restriction to ground terms may be removed in case the stream specification is data independent: left hand sides of rules do not contain data values. In Section 6 we discuss fixpoints and prove incompleteness. We conclude in Section 7.

\section{Streams: Specifications and Models}

In stream specifications we have two sorts: $s$ (stream) and $d$ (data). We assume the set $D$ of data elements to consist of the unique normal forms of ground terms over some signature $\Sigma_{d}$ with respect to some terminating orthogonal rewrite system $R_{d}$ over $\Sigma_{d}$. Here all symbols of $\Sigma_{d}$ are of type $d^{n} \rightarrow d$ for some $n \geq 0$. We assume a particular symbol : having type $d \times s \rightarrow s$. For giving the actual stream specification we need a set $\Sigma_{s}$ of stream symbols, each being of type $d^{n} \times s^{m} \rightarrow s$ for $n, m \geq 0$. Now terms of sort $s$ are defined inductively as follows:

- a variable of sort $s$ is a term of sort $s$,

- if $f \in \Sigma_{s}$ is of type $d^{n} \times s^{m} \rightarrow s, u_{1}, \ldots, u_{n}$ are terms over $\Sigma_{d}$ and $t_{1}, \ldots, t_{m}$ are terms of sort $s$, then $f\left(u_{1}, \ldots, u_{n}, t_{1}, \ldots, t_{m}\right)$ is a term of sort $s$,

- if $u$ is a term over $\Sigma_{d}$ and $t$ is a term of sort $s$, then $u: t$ is a term of sort $s$.

As a notational convention variables of sort $d$ will be denoted by $x, y$, terms of sort $d$ by $u, u_{i}$, variables of sort $s$ by $\sigma, \tau$, and terms of sort $s$ by $t, t_{i}$.

Definition 1. A stream specification $\left(\Sigma_{d}, \Sigma_{s}, R_{d}, R_{s}\right)$ consists of $\Sigma_{d}, \Sigma_{s}, R_{d}$ as given before, and a set $R_{s}$ of rewrite rules of the shape

$$
f\left(u_{1}, \ldots, u_{n}, t_{1}, \ldots, t_{m}\right) \rightarrow t
$$

where

$-f \in \Sigma_{s}$ is of type $d^{n} \times s^{m} \rightarrow s$,

- for every $i=1, \ldots, n$ the term $u_{i}$ is either a variable of sort $d$ or $u_{i} \in D$,

- for every $i=1, \ldots, m$ the term $t_{i}$ is either a variable of sort $s$, or $t_{i}=x: \sigma$ where $x$ is a variable of sort $d$ and $\sigma$ is a variable of sort $s$,

- $t$ is any term of sort $s$,

- every ground term of sort $s$ being in normal form with respect to $R_{d}$ matches with the left hand side of exactly one rule from $R_{s}$. 
Due to these requirements $R_{s} \cup R_{d}$ is orthogonal. Sometimes we call $R_{s}$ a stream specification: in that case $\Sigma_{d}, \Sigma_{s}$ consist of the symbols of sort $d, s$, respectively, occurring in $R_{s}$, and $R_{d}=\emptyset$. Rules $\ell \rightarrow r$ in $R_{s}$ are often written as $\ell=r$.

Example 1. For specifying the Thue Morse sequence the data elements are 0,1, and a data operation not is used. The data rewrite system $R_{d}$ consists of the two rules not $(0) \rightarrow 1$ and $\operatorname{not}(1) \rightarrow 0$. The rewrite system $R_{s}$ consists of the rules

$$
\begin{aligned}
\text { morse } & \rightarrow 0: \operatorname{zip}(\operatorname{inv}(\text { morse) }) \text { tail(morse) }) & \operatorname{tail}(x: \sigma) & \rightarrow \sigma \\
\operatorname{inv}(x: \sigma) & \rightarrow \operatorname{not}(x): \operatorname{inv}(\sigma) & \operatorname{zip}(x: \sigma, \tau) & \rightarrow x: \operatorname{zip}(\tau, \sigma)
\end{aligned}
$$

Definition 1 is closely related to the definition of stream specification in [3]. In fact there are two differences:

- We want to specify streams for every ground term of sort $s$, while in [3] there is a designated constant to be specified.

- The restriction on left hand sides is stronger than the exhaustiveness from [3]. However, by introducing fresh symbols and rules for defining these fresh symbols, every stream specification in the format of [3] can be unfolded to a stream specification in our format.

For instance, the function $f$ in the introduction to define the Fibonacci stream does not meet our requirements since the argument $0: \sigma$ in the left hand side $f(0: \sigma)$ is not of the right shape. Introducing a fresh symbol $g$ and unfolding yields

$$
\begin{aligned}
& f(x: \sigma)=g(x, \sigma) \quad g(0, \sigma)=0: 1: f(\sigma) \\
& g(1, \sigma)=0: f(\sigma)
\end{aligned}
$$

satisfying our format.

Stream specifications are intended to specify streams for the constants in $\Sigma_{s}$, and stream functions for the other elements of $\Sigma_{s}$. The combination of these streams and stream functions is what we will call a stream model.

More precisely, a stream over $D$ is a map from the natural numbers to $D$. Write $D^{\omega}$ for the set of all streams over $D$. In case of $D=\emptyset$ we have $D^{\omega}=\emptyset$; in case of $\# D=1$ we have $\# D^{\omega}=1$. So in non-degenerate cases we have $\# D \geq 2$.

It seems natural to require that stream functions in a stream model are defined on all streams. However, it turns out that several desired properties do not hold when requiring this. Therefore we allow stream functions to be defined on some set $S \subseteq D^{\omega}$ for which every ground term can be interpreted in $S$.

Definition 2. A stream model is defined to consist of a set $S \subseteq D^{\omega}$ and a set of functions $[f]$ for every $f \in \Sigma_{s}$, where $[f]: D^{n} \times S^{m} \rightarrow S$ if the type of $f$ is $d^{n} \times s^{m} \rightarrow s$.

For a ground term $u$ over $\Sigma_{d}$ write $\mathbf{N F}(u)$ for its $R_{d}$-normal form. For $f \in \Sigma_{d}$ and $u_{1}, \ldots, u_{n} \in D$ we define $\left[f\left(u_{1}, \ldots, u_{n}\right)\right]=\mathbf{N F}\left(f\left(u_{1}, \ldots, u_{n}\right)\right)$. We write $\mathcal{T}_{s}$ 
for the set of ground terms of sort $s$. For $t \in \mathcal{T}_{s}$ the stream interpretation $[t]$ in the stream model $\left(S,([f])_{f \in \Sigma_{s}}\right)$ is defined inductively by:

$$
\begin{aligned}
{\left[f\left(u_{1}, \ldots, u_{n}, t_{1}, \ldots, t_{m}\right)\right] } & =[f]\left(\left[u_{1}\right], \ldots,\left[u_{n}\right],\left[t_{1}\right], \ldots,\left[t_{m}\right]\right) & & \text { for } f \in \Sigma_{s} \\
{[u: t](0) } & =[u] & & \\
{[u: t](i) } & =[t](i-1) & & \text { for } i>0
\end{aligned}
$$

for all ground terms $u, u_{i}$ of sort $d$ and all ground terms $t, t_{i}$ of sort $s$.

So in a stream model:

- every data operator is interpreted by its corresponding term constructor, after which the result is reduced to normal form,

- every stream operator $f$ is interpreted by the given function $[f]$, and

- the operator : applied on a data element $d$ and a stream $s$ is interpreted by putting $d$ on the first position and shifting every stream element of $s$ to its next position.

Definition 3. A stream model $\left(S,([f])_{f \in \Sigma_{s}}\right)$ is said to satisfy a stream specification $\left(\Sigma_{d}, \Sigma_{s}, R_{d}, R_{s}\right)$ if $[\ell \rho]=[r \rho]$ for every rule $\ell \rightarrow r$ in $R_{s}$ and every ground substitution $\rho$. We also say that the specification admits the model.

Now we can express the desired well-definedness of a stream specification more precisely: there is exactly one stream model $\left(S,([f])_{f \in \Sigma_{s}}\right)$ satisfying the stream specification for which $S=\left\{[t] \mid t \in \mathcal{T}_{s}\right\}$. This is not always the case: if $\# D>1$ and $R_{s}$ consists of the rule $c \rightarrow c$ there is not a unique $[c]$ since every stream satisfies the specification. Less trivial is the boolean stream specification

$$
c=0: f(c), \quad f(x: \sigma)=\sigma,
$$

in which $[f]$ can be chosen to be the tail function and $[c]$ be any stream starting with 0 , showing non-uniqueness of stream models.

\section{The Observational Variant}

In this paper we define a transformation Obs transforming the original TRS $R_{s}$ to its observational variant $\operatorname{Obs}\left(R_{s}\right)$. The basic idea is that the streams are observed by two auxiliary operator head and tail, of which head picks the first element of the stream and tail removes the first element from the stream, and that for every $t \in \mathcal{T}_{s}$ of type stream both head $(t)$ and tail $(t)$ can be rewritten by $\operatorname{Obs}\left(R_{s}\right)$.

The main result of this paper is that if $\operatorname{Obs}\left(R_{s}\right) \cup R_{d}$ is terminating for a given specification $\left(\Sigma_{d}, \Sigma_{s}, R_{d}, R_{s}\right)$, then it admits a unique model $\left(S,([f])_{f \in \Sigma_{s}}\right)$ satisfying $S=\left\{[t] \mid t \in \mathcal{T}_{s}\right\}$. As a consequence, the specification uniquely defines a corresponding stream $[t]$ for every $t \in \mathcal{T}_{s}$.

We define $\operatorname{Obs}\left(R_{s}\right)$ in two steps. First we define $\mathrm{P}\left(R_{s}\right)$ obtained from $R_{s}$ by modifying the rules as follows. By definition every rule of $R_{s}$ is of the shape

$$
f\left(u_{1}, \ldots, u_{n}, t_{1}, \ldots, t_{m}\right) \rightarrow t
$$


where for every $i=1, \ldots, m$ the term $t_{i}$ is either a variable of sort $s$, or $t_{i}=x: \sigma$ where $x$ is a variable of sort $d$ and $\sigma$ is a variable of sort $s$. In case $t_{i}=x: \sigma$ then in the left hand side of the rule the subterm $t_{i}$ is replaced by $\sigma$, while in the right hand side of the rule every occurrence of $x$ is replaced by head $(\sigma)$ and every occurrence of $\sigma$ is replaced by $\operatorname{tail}(\sigma)$.

For example, the zip rule in Example 1 will be replaced by

$$
\operatorname{zip}(\sigma, \tau) \rightarrow \operatorname{head}(\sigma): \operatorname{zip}(\tau, \operatorname{tail}(\sigma)) .
$$

Now we are ready to define Obs.

Definition 4. Let $\left(\Sigma_{d}, \Sigma_{s}, R_{d}, R_{s}\right)$ be a stream specification. Let $\mathrm{P}\left(R_{s}\right)$ be defined as above. Then $\operatorname{Obs}\left(R_{s}\right)$ is the TRS over $\Sigma_{d} \cup \Sigma_{s} \cup\{$ :, head, tail $\}$ consisting of

- the two rules

$$
\operatorname{head}(x: \sigma) \rightarrow x, \quad \operatorname{tail}(x: \sigma) \rightarrow \sigma,
$$

- for every rule in $\mathrm{P}\left(R_{s}\right)$ of the shape $\ell \rightarrow u: t$ the two rules

$$
\operatorname{head}(\ell) \rightarrow u, \quad \operatorname{tail}(\ell) \rightarrow t,
$$

- for every rule in $\mathrm{P}\left(R_{s}\right)$ of the shape $\ell \rightarrow r$ with $\operatorname{root}(r) \neq$ : the two rules

$$
\operatorname{head}(\ell) \rightarrow \operatorname{head}(r), \quad \operatorname{tail}(\ell) \rightarrow \operatorname{tail}(r) .
$$

Example 2. For the TRS $R_{s}$ given in Example 1 we rename the symbol tail by tail0 in order to keep the symbol tail for the fresh symbol introduced in the Obs construction. Then the TRS $\operatorname{Obs}\left(R_{s}\right)$ consists of the following rules:

$$
\begin{array}{rlrl}
\operatorname{head}(x: \sigma) & \rightarrow x & \operatorname{head}(\operatorname{tail} 0(\sigma)) & \rightarrow \text { head }(\operatorname{tail}(\sigma)) \\
\operatorname{tail}(x: \sigma) & \rightarrow \sigma & \operatorname{tail}(\operatorname{tail}(\sigma)) & \rightarrow \operatorname{tail}(\operatorname{tail}(\sigma)) \\
\operatorname{head}(\operatorname{morse}) & \rightarrow 0 & \operatorname{tail}(\operatorname{zip}(\sigma, \tau)) & \rightarrow \operatorname{head}(\sigma) \\
\operatorname{tail}(\operatorname{morse}) & \rightarrow \operatorname{zip}(\operatorname{inv}(\operatorname{morse}), \operatorname{tail}(\operatorname{morse})) & \\
\operatorname{head}(\operatorname{inv}(\sigma)) & \rightarrow \operatorname{not}(\operatorname{tail}(\sigma)) \\
\operatorname{tail}(\operatorname{inv}(\sigma)) & \rightarrow \operatorname{inv}(\operatorname{tail}(\sigma)) &
\end{array}
$$

Together with the rules not $(0) \rightarrow 1$ and not $(1) \rightarrow 0$ from $R_{d}$ this TRS is terminating as can easily be proved fully automatically by AProVE [4] or TTT2 [7]. As a consequence, the result of this paper states that the specification uniquely defines a stream for every ground term of type $s$, in particular for morse.

\section{The Main Theorem}

We start this section by presenting our main theorem.

Theorem 1. Let $\left(\Sigma_{d}, \Sigma_{s}, R_{d}, R_{s}\right)$ be a stream specification for which the TRS $\operatorname{Obs}\left(R_{s}\right) \cup R_{d}$ is terminating. Then the stream specification admits a unique model $\left(S,([f])_{f \in \Sigma_{s}}\right)$ satisfying $S=\left\{[t] \mid t \in \mathcal{T}_{s}\right\}$. 
Before proving the theorem we show by an example why it is essential to restrict to $S=\left\{[t] \mid t \in \mathcal{T}_{s}\right\}$ rather than choosing $S=D^{\omega}$. A degenerate example is obtained if there are no constants of sort $s$, and hence $\mathcal{T}_{s}=\emptyset$. More interesting is the following.

Example 3. Let the boolean stream specification consist of $R_{d}=\emptyset$ and $R_{s}$ consisting of the following rules:

$$
\begin{array}{rlrl}
c & \rightarrow 1: c & g(0, \sigma) & \rightarrow f(\sigma) \\
f(x: \sigma) & \rightarrow g(x, \sigma) & g(1, \sigma) & \rightarrow 1: f(\sigma)
\end{array}
$$

So $f$ tries to remove all 0's from its argument. For streams containing infinitely many 0's this may be problematic. Note that by the symbols $c,:, 0$ and 1 only the streams with finitely many 0 's can be constructed, for ground terms this problem does not arise. Indeed the TRS $\operatorname{Obs}\left(R_{s}\right) \cup R_{d}$ is terminating, and by Theorem 1 the specification admits a unique model $\left(S,([f])_{f \in \Sigma_{s}}\right)$ satisfying $S=\{[t] \mid t \in$ $\left.\mathcal{T}_{s}\right\}$. However, when extending to all streams the function $[f]: D^{\omega} \rightarrow D^{\omega}$ is not uniquely defined, even if we strengthen the requirement of $[\ell \rho]=[r \rho]$ for all rules $\ell \rightarrow r$ and all ground substitutions $\rho$ to an open variant in which the $\sigma$ 's in the rules are replaced by arbitrary streams. Write ones and zeros for the streams only consisting of ones, resp. zeros. Two distinct models $[\cdot]_{1}$ and $[\cdot]_{2}$ satisfying the stream specification are defined by:

$$
[c]_{1}=[f]_{1}(s)=[g]_{1}(u, s)=\text { ones for all } s \in D^{\omega}, u \in D,
$$

and $[c]_{2}=[f]_{2}(s)=[g]_{2}(u, s)=$ ones for $u \in D$ and streams $s$ containing infinitely many ones, and $[f]_{2}(s)=1^{n}$ : zeros, $[g]_{2}(u, s)=[f]_{2}(u: s)$ for $u \in D$ and streams $s$ containing $n<\infty$ ones.

Now we arrive at the proof of Theorem 1. The plan of the proof is as follows.

- First we construct a function $[\cdot]_{1}: \mathcal{T}_{s} \rightarrow D^{\omega}$, and choose $S_{1}=\left\{[t]_{1} \mid t \in \mathcal{T}_{s}\right\}$.

- Next we show that if $\left[t_{i}\right]_{1}=\left[t_{i}^{\prime}\right]_{1}$ for $i=1, \ldots, m$, then

$$
\left[f\left(u_{1}, \ldots, u_{n}, t_{1}, \ldots, t_{m}\right)\right]_{1}=\left[f\left(u_{1}, \ldots, u_{n}, t_{1}^{\prime}, \ldots, t_{m}^{\prime}\right)\right]_{1},
$$

by which $[f]_{1}$ is well-defined and we have a model $\left(S_{1},\left([f]_{1}\right)_{f \in \Sigma_{s}}\right)$.

- We show this model satisfies the specification.

- We show no other model $\left(S,([f])_{f \in \Sigma_{s}}\right)$ satisfies the specification and $S=$ $\left\{[t] \mid t \in \mathcal{T}_{s}\right\}$.

First we define $[t]_{1} \in D^{\omega}$ for any $t \in \mathcal{T}_{s}$. Since elements of $D^{\omega}$ are functions from $\mathbf{N}$ to $D$, a function $[t]_{1} \in D^{\omega}$ is defined by defining $[t]_{1}(n)$ for every $n \in \mathbf{N}$. Due to the assumption of the theorem the TRS $\operatorname{Obs}\left(R_{s}\right) \cup R_{d}$ is terminating. According to the definition of stream specification the TRS $R_{s} \cup R_{d}$ is orthogonal, and by the construction Obs the TRS $\operatorname{Obs}\left(R_{s}\right) \cup R_{d}$ is orthogonal too. So every ground term of sort $d$ has a unique normal form with respect to $\operatorname{Obs}\left(R_{s}\right) \cup R_{d}$.

Assume such a normal form contains a symbol from $\Sigma_{s} \cup\{:\}$. Choose such a symbol with minimal position, that is, closest to the root. Since the term is 
of sort $d$, this symbol is not the root. Hence it has a parent. Due to minimality of position, this parent is either head or tail. Due to the shape of the rules of $\operatorname{Obs}\left(R_{s}\right)$, a rule of $\operatorname{Obs}\left(R_{s}\right)$ is applicable on this parent position, contradicting the normal form assumption. So the normal form only contains symbols from $\Sigma_{d}$. Since it is also a normal form with respect to $R_{d}$, such a normal form is an element of $D$. Now for $t \in \mathcal{T}_{s}$ and $n \in \mathbf{N}$ we define

$$
[t]_{1}(n)=\text { the normal form of head }\left(\operatorname{tail}^{n}(t)\right) \text { with respect to } \operatorname{Obs}\left(R_{s}\right) \cup R_{d},
$$

in this way defining $[t]_{1} \in D^{\omega}$.

Lemma 1. Let $\operatorname{Obs}\left(R_{s}\right) \cup R_{d}$ be terminating. Let $f \in \Sigma_{s}$ of type $d^{n} \times s^{m} \rightarrow s$. Let $u_{1}, \ldots, u_{n} \in D$ and $t_{1}, \ldots, t_{m}, t_{1}^{\prime}, \ldots, t_{m}^{\prime} \in \mathcal{T}_{s}$ satisfying $\left[t_{i}\right]_{1}=\left[t_{i}^{\prime}\right]_{1}$ for $i=1, \ldots, m$. Then

$$
\left[f\left(u_{1}, \ldots, u_{n}, t_{1}, \ldots, t_{m}\right)\right]_{1}=\left[f\left(u_{1}, \ldots, u_{n}, t_{1}^{\prime}, \ldots, t_{m}^{\prime}\right)\right]_{1} .
$$

Proof. First we extend the definition of $[\cdot]_{1}$ to all ground terms over $\Sigma_{s} \cup \Sigma_{d} \cup\{$ : , head, tail $\}$. For ground terms $t$ of sort $s$ we define it by $[t]_{1}(n)=$ the normal form of head( $\left.\operatorname{tail}^{n}(t)\right)$ with respect to $\operatorname{Obs}\left(R_{s}\right) \cup R_{d}$, and for ground terms $u$ of sort $d$ we define $[u]_{1}$ to be the normal form of $u$ with respect to $\operatorname{Obs}\left(R_{s}\right) \cup R_{d}$. We prove the following claim.

Claim 1: Let $[t]_{1}=\left[t^{\prime}\right]_{1}$ for $t, t^{\prime} \in \mathcal{T}_{s}$. Let $T$ be a ground term over $\Sigma_{s} \cup \Sigma_{d} \cup\{:$, head, tail $\}$ of sort $s$ containing $t$ as a subterm. Let $T^{\prime}$ be obtained from $T$ by replacing zero or more occurrences of the subterm $t$ by $t^{\prime}$. Then

$$
[\operatorname{head}(T)]_{1}=\left[\operatorname{head}\left(T^{\prime}\right)\right]_{1} .
$$

Let $>$ be the well-founded order on ground terms being the strict part of $\geq$ defined by

$$
v \geq v^{\prime} \Longleftrightarrow v^{\prime} \text { is a subterm } v^{\prime \prime} \text { such that } v \rightarrow_{\mathrm{Obs}\left(R_{s}\right) \cup R_{d}}^{*} v^{\prime \prime} .
$$

We prove the claim for every such term head $(T)$ by noetherian induction on $>$.

Claim 1 is trivial if $t=T$, so we may assume that $T=f\left(u_{1}, \ldots, u_{n}, t_{1}, \ldots, t_{m}\right)$ such that $t$ occurs in $u_{1}, \ldots, u_{n}, t_{1}, \ldots, t_{m}$, and either $f \in \Sigma_{s} \cup\{:$, tail $\}$, and $T^{\prime}=f\left(u_{1}^{\prime}, \ldots, u_{n}^{\prime}, t_{1}^{\prime}, \ldots, t_{m}^{\prime}\right)$. For every subterm of $u_{i}$ of the shape head $(\cdots)$ we may apply the induction hypothesis, yielding $\left[u_{i}\right]_{1}=\left[u_{i}^{\prime}\right]_{1}=d_{i}$ for all $i$, defining $d_{i} \in D$.

In case the root of $T$ is not tail we rewrite

$$
\operatorname{head}(T) \rightarrow{ }^{*} \operatorname{Obs}\left(R_{s}\right) \cup R_{d} \operatorname{head}\left(f\left(d_{1}, \ldots, d_{n}, t_{1}, \ldots, t_{m}\right)\right.
$$

by the rule head $(f(\cdots)) \rightarrow \cdots$ in $\operatorname{Obs}\left(R_{s}\right)$, yielding a term $U$ of sort $d$. The only way such a term can contain $t$ as a subterm is by $U=C\left[\operatorname{head}\left(V_{1}\right), \ldots, \operatorname{head}\left(V_{k}\right)\right]$ where $t$ is a subterm of some of the $V_{i}$ and $C$ is composed from $\Sigma_{d}$. By the induction hypothesis we obtain $\left[\operatorname{head}\left(V_{i}\right)\right]_{1}=\left[\operatorname{head}\left(V_{i}^{\prime}\right)\right]_{1}$ for $V_{i}^{\prime}$ obtained from $V_{i}$ by 
replacing zero ore more occurrences of $t$ by $t^{\prime}$. Hence $[\operatorname{head}(T)]_{1}=$ $\left.\left.C\left[\operatorname{head}\left(V_{1}\right), \ldots, \operatorname{head}\left(V_{k}\right)\right]\right]_{1}=C\left[\operatorname{head}\left(V_{1}^{\prime}\right), \ldots, \operatorname{head}\left(V_{k}^{\prime}\right)\right]\right]_{1}=\left[\operatorname{head}\left(T^{\prime}\right)\right]_{1}$.

In case the root of $T$ is tail then write $T=\operatorname{tail}^{i}(f(\cdots)) \rightarrow^{*} \operatorname{Obs}\left(R_{s}\right) \cup R_{d}$ tail $^{i}\left(f\left(d_{1}, \ldots, d_{n}, t_{1}, \ldots, t_{m}\right)\right.$ for $f \in \Sigma_{s} \cup\{:\}$. This can be rewritten by the rule tail $(f(\cdots)) \rightarrow \cdots$ in $\operatorname{Obs}\left(R_{s}\right)$, yielding $V$. On the same position using the same rule we can rewrite $T^{\prime} \rightarrow \operatorname{Obs}\left(R_{s}\right) V^{\prime}$ for $V^{\prime}$ obtained from $V$ by replacing one ore more occurrences of $t$ by $t^{\prime}$. Applying the induction hypothesis gives $[\operatorname{head}(V)]_{1}=\left[\operatorname{head}\left(V^{\prime}\right)\right]_{1}$ yielding

$$
[\operatorname{head}(T)]_{1}=[\operatorname{head}(V)]_{1}=\left[\operatorname{head}\left(V^{\prime}\right)\right]_{1}=\left[\operatorname{head}\left(T^{\prime}\right)\right]_{1},
$$

concluding the proof of Claim 1.

Claim 2: Let $[t]_{1}=\left[t^{\prime}\right]_{1}$ for $t, t^{\prime} \in \mathcal{T}_{s}$. Let $T$ be a ground term over $\Sigma_{s} \cup \Sigma_{d} \cup\{$ :, head, tail $\}$ of sort $s$ containing $t$ as a subterm. Let $T^{\prime}$ be obtained from $T$ by replacing one or more occurrences of the subterm $t$ by $t^{\prime}$. Then $[T]_{1}=\left[T^{\prime}\right]_{1}$.

Claim 2 easily follows from Claim 1 and the observation

$$
[T]_{1}=\left[T^{\prime}\right]_{1} \Longleftrightarrow \forall i \in \mathbf{N}:\left[\operatorname{head}\left(\operatorname{tail}^{i}(T)\right]_{1}=\left[\operatorname{head}\left(\operatorname{tail}^{i}\left(T^{\prime}\right)\right]_{1} .\right.\right.
$$

Now the lemma follows by applying Claim 2 and replacing $t_{i}$ by $t_{i}^{\prime}$ successively for $i=1, \ldots, m$.

Define $S_{1}=\left\{[t]_{1} \mid t \in \mathcal{T}_{s}\right\}$. For any $f \in \Sigma_{s}$ of type $d^{n} \times s^{m} \rightarrow s$ for $u_{1}, \ldots, u_{n} \in D$ and $t_{1}, \ldots, t_{m}, t_{1}^{\prime}, \ldots, t_{m}^{\prime} \in \mathcal{T}_{s}$ we now define $[f]_{1}: D^{n} \times S^{m} \rightarrow S$ by

$$
[f]_{1}\left(u_{1}, \ldots, u_{n},\left[t_{1}\right], \ldots,\left[t_{m}\right]\right)=\left[f\left(u_{1}, \ldots, u_{n}, t_{1}, \ldots, t_{m}\right)\right]_{1} ;
$$

Lemma 1 implies that this is well-defined: the result is independent of the choice of the representants in $\left[t_{i}\right]_{1}$. So $\left(S_{1},\left([f]_{1}\right)_{f \in \Sigma_{s}}\right)$ is a model.

Next we will prove that it satisfies the specification, and essentially is the only one doing so.

Lemma 2. Let $\ell \rightarrow r \in R_{s}$ and let $\rho$ be a substitution. Then

- there is a term $t$ such that head $(\ell \rho) \rightarrow_{\mathrm{Obs}\left(R_{s}\right)}^{*} t$ and head $(r \rho) \rightarrow \stackrel{*}{\mathrm{Obs}\left(R_{s}\right)} t$, and

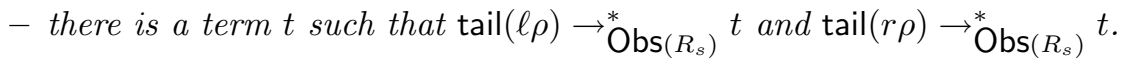

Proof. Let $f$ be the root of $\ell$. Define $\rho^{\prime}$ by $\sigma \rho^{\prime}=x \rho: \sigma \rho$ for every argument of the shape $x: \sigma$ of $f$ in $\ell$, and $\rho^{\prime}$ coincides with $\rho$ on all other variables. Then head $(\ell \rho)=\ell^{\prime} \rho^{\prime}$ for some rule in $\ell^{\prime} \rightarrow r^{\prime}$ in $\operatorname{Obs}\left(R_{s}\right)$. Now a common reduct $t$ of $r^{\prime} \rho^{\prime}$ and head $(r \rho)$ is obtained by applying the $\operatorname{rule} \operatorname{head}(x: \sigma) \rightarrow x$ zero or more times. This yields head $(\ell \rho)=\ell^{\prime} \rho^{\prime} \rightarrow \operatorname{Obs}\left(R_{s}\right) r^{\prime} \rho^{\prime} \rightarrow{ }_{\operatorname{Obs}\left(R_{s}\right)}^{*} t$ and

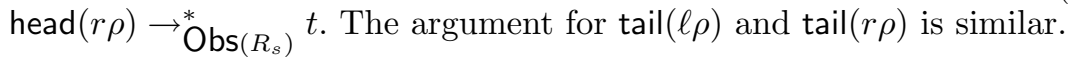


Lemma 3. The model $\left(S_{1},\left([f]_{1}\right)_{f \in \Sigma_{s}}\right)$ satisfies the specification $\left(\Sigma_{d}, \Sigma_{s}, R_{d}, R_{s}\right)$.

Proof. We have to prove that $[\ell \rho]_{1}(i)=[r \rho]_{1}(i)$ for every rule $\ell \rightarrow r$ in $R_{s}$, every ground substitution $\rho$ and every $i \in \mathbf{N}$. By definition $[\ell \rho]_{1}(i)$ is the unique normal form with respect to $\operatorname{Obs}\left(R_{s}\right) \cup R_{d}$ of head $\left(\operatorname{tail} i^{i}(\ell \rho)\right)$, and $[r \rho]_{1}(i)$ is the similar normal form of head $\left(\operatorname{tail}^{i}(r \rho)\right)$. Now the lemma follows from Lemma 2.

For concluding the proof of Theorem 1 we have to prove that $\left(S_{1},\left([f]_{1}\right)_{f \in \Sigma_{s}}\right)$ is the only model satisfying the specification $\left(\Sigma_{d}, \Sigma_{s}, R_{d}, R_{s}\right)$ and $S=\{[t] \mid t \in$ $\left.\mathcal{T}_{s}\right\}$. This follows from the following lemma.

Lemma 4. Let $\left(S,([f])_{f \in \Sigma_{s}}\right)$ be any model satisfying $\left(\Sigma_{d}, \Sigma_{s}, R_{d}, R_{s}\right)$, and $t \in$ $\mathcal{T}_{s}$. Then $[t]=[t]_{1}$.

Proof. By definition in the model for $u \in D$ and $s \in S$ we have

$$
([:](u, s))(0)=u, \quad([:](u, s))(i)=s(i-1) \text { for } i>0 .
$$

In the original stream specification the symbols head, tail do not occur, for these fresh symbols we now define functions [head] and [tail] on streams $s$ by

$$
[\text { head }](s)=s(0), \quad([\text { tail }](s))(i)=s(i+1) \text { for } i \geq 0 .
$$

If $S \neq D^{\omega}$ then it is not clear whether [tail] $(s) \in S$ for every $s \in S$. Therefore we extend $S$ to $D^{\omega}$ and define $[f](\cdots)$ to be any arbitrary value if at least one argument is in $D^{\omega} \backslash S$; note that for the model satisfying the specification we only required $[\ell \rho]=[r \rho]$ for ground substitutions to $\mathcal{T}_{s}$ by which these junk values do not play a role.

Due to the definitions of [:], [head] and [tail] this extended model satisfies the equations

$$
\mathcal{E}=\left\{\begin{aligned}
\operatorname{head}(x: \sigma) & =x \\
\operatorname{tail}(x: \sigma) & =\sigma \\
\sigma & =\operatorname{head}(\sigma): \operatorname{tail}(\sigma)
\end{aligned}\right.
$$

that is, for $\rho$ mapping $x$ to any term of sort $d$ and $\sigma$ to any term of sort $s$ we have $[\ell \rho]=[r \rho]$ for every $\ell \rightarrow r \in \mathcal{E}$. From the definition of $\operatorname{Obs}\left(R_{s}\right)$ it is easily checked that any innermost step $t \rightarrow \operatorname{Obs}\left(R_{s}\right) t^{\prime}$ on a ground term $t$ is either an application of one of the first two rules of $\mathcal{E}$, or it is of the shape

$$
t \rightarrow_{\mathcal{E}}^{*} \cdot \rightarrow_{R_{s}} \cdot \rightarrow_{\mathcal{E}}^{*} t^{\prime}
$$

where due to the innermost requirement the redex of the $\rightarrow_{R_{s}}$ step does not contain the symbols head or tail so is in $\mathcal{T}_{s}$. Since the model is assumed to satisfy the specification $\left(\Sigma_{d}, \Sigma_{s}, R_{d}, R_{s}\right)$, we conclude that $[t]=\left[t^{\prime}\right]$ for every innermost ground step $t \rightarrow \mathrm{Obs}\left(R_{s}\right) t^{\prime}$.

For the lemma we have to prove that $[t](i)=[t]_{1}(i)$ for every $i \in \mathbf{N}$. By definition $[t]_{1}(i)$ is the normal form with respect to $\operatorname{Obs}\left(R_{s}\right) \cup R_{d}$ of head(tail $\left.{ }^{i}(t)\right)$. 
Now consider an innermost $\operatorname{Obs}\left(R_{s}\right) \cup R_{d}$-reduction of head $\left(\operatorname{tail}^{i}(t)\right)$ to $[t]_{1}(i)$. By the above observation and the definitions of [head] and [tail] we conclude that

$$
[t](i)=\left[\operatorname{head}\left(\operatorname{tail}^{i}(t)\right)\right]=\left[[t]_{1}(i)\right]=[t]_{1}(i),
$$

the last step since $[t]_{1}(i) \in D$. This concludes the proof, both of the lemma and Theorem 1.

We conclude this section by an example of a well-defined stream specification that is not productive.

Example 4. Choose $\Sigma_{s}=\{c, f, g\}, \Sigma_{d}=\{0,1\}, R_{d}=\emptyset$, and $R_{s}$ consists of the following rules:

$$
\begin{aligned}
c & =1: c \\
f(x: \sigma) & =g(f(\sigma)) \\
g(x: \sigma) & =c .
\end{aligned}
$$

Then this is a valid stream specification for which $\operatorname{Obs}\left(R_{s}\right)$ is terminating, as can be shown by AProVE [4] or TTT2 [7]. Hence by Theorem 1 there is a unique model. So the ground term $f(c)$ has a unique interpretation: the stream only consisting of 1's. However, $f(c)$ is not productive.

So the TRS $R_{s}$ is not suitable to compute the interpretation of $f(c)$. Instead one can use outermost reduction with respect to $\mathrm{P}\left(R_{s}\right)$, where $\mathrm{P}\left(R_{s}\right)$ is the TRS introduced in the definition of $\operatorname{Obs}\left(R_{s}\right)$.

\section{Data Independent Stream Functions}

The reason that in Theorem 1 we have to restrict to models satisfying $S=$ $\left\{[t] \mid t \in \mathcal{T}_{s}\right\}$, as we saw in Example 3, is in the fact that computations may be guarded by data elements in left hand sides of rules. Next we show that we also get well-definedness for stream functions defined on all streams in case the left hand sides of the rule do not contain data elements.

Theorem 2. Let $\left(\Sigma_{d}, \Sigma_{s}, R_{d}, R_{s}\right)$ be a stream specification for which the TRS $\operatorname{Obs}\left(R_{s}\right) \cup R_{d}$ is terminating and the only subterms of left hand sides of $R_{s}$ of sort $d$ are variables. Then the stream specification admits a unique model $\left(S,([f])_{f \in \Sigma_{s}}\right)$ satisfying $S=D^{\omega}$.

Proof. (sketch) We have to prove that for any $f \in \Sigma_{s}$ of type $d^{n} \times s^{m} \rightarrow s$ the function $[f]: D^{n} \times\left(D^{\omega}\right)^{m} \rightarrow D^{\omega}$ is uniquely defined. For doing so we introduce $m$ fresh constants $c_{1}, \ldots, c_{m}$ of sort $s$. Let $k \in \mathbf{N}$ and $u_{1}, \ldots, u_{n} \in D$. Due to termination and orthogonality of $\operatorname{Obs}\left(R_{s}\right) \cup R_{d}$, the term

$$
\operatorname{head}\left(\operatorname{tail}^{k}\left(f\left(u_{1}, \ldots, u_{n}, c_{1}, \ldots, c_{m}\right)\right)\right)
$$

has a unique normal for with respect to $\operatorname{Obs}\left(R_{s}\right) \cup R_{d}$. Since it is of sort $d$, due to the shape of the rules it is a ground term of sort $d$ over $\Sigma_{d} \cup\left\{\right.$ head, tail, $\left.c_{1}, \ldots, c_{m}\right\}$, that is, a ground term $T$ composed from $\Sigma_{d}$ and terms of the shape head $\left(\operatorname{tail}{ }^{i}\left(c_{j}\right)\right)$ 
for $i \in \mathbf{N}$ and $j \in\{1, \ldots, m\}$. For this observation it is essential that left hand sides do not contain non-variable terms of sort $d$ : terms of the shape $f($ head $(\cdots), \ldots)$ should be rewritten.

Let $N$ be the greatest number $i$ for which $T$ has a subterm of the shape head $\left(\operatorname{tail}^{i}\left(c_{j}\right)\right)$. Let $s_{1}, \ldots, s_{m} \in D^{\omega}$. Define $t_{j}=s_{j}(0): s_{j}(1): \cdots: s_{j}(N): \sigma$. Since head $\left(\operatorname{tail}^{k}\left(f\left(u_{1}, \ldots, u_{n}, c_{1}, \ldots, c_{m}\right)\right)\right)$ rewrites to $T$, the term head $\left(\operatorname{tail}^{k}\left(f\left(u_{1}, \ldots, u_{n}, t_{1}, \ldots, t_{m}\right)\right)\right)$ rewrites to $T^{\prime}$ obtained from $T$ by replacing every subterm of the shape head $\left(\operatorname{tail}^{i}\left(c_{j}\right)\right)$ by head $\left(\operatorname{tail}^{i}\left(t_{j}\right)\right)$. Observe that head $\left(\operatorname{tail}^{i}\left(t_{j}\right)\right)$ rewrites to $s_{j}(i) \in D$. So $\left([f]\left(u_{1}, \ldots, u_{n}, s_{1}, \ldots, s_{m}\right)\right)(k)$ has to be the $R_{d}$-normal form of the ground term over $\Sigma_{d}$ obtained from $T$ by replacing every subterm of the shape head $\left(\operatorname{tail}^{i}\left(c_{j}\right)\right)$ by $s_{j}(i) \in D$. Since this fixes $\left([f]\left(u_{1}, \ldots, u_{n}, s_{1}, \ldots, s_{m}\right)\right)(k)$ for every $k$, this uniquely defines $[f]$.

Example 5. It is easy to see that for the standard stream functions zip, even and odd defined by

$$
\operatorname{even}(x: \sigma)=x: \operatorname{odd}(\sigma), \operatorname{odd}(x: \sigma)=\operatorname{even}(\sigma), \quad \operatorname{zip}(x: \sigma, \tau)=x: \operatorname{zip}(\tau, \sigma),
$$

there exists $f: D^{\omega} \rightarrow D^{\omega}$ for every data set $D$ satisfying

$$
f(x: \sigma)=x: \operatorname{zip}(f(\operatorname{even}(\sigma)), f(\operatorname{odd}(\sigma))),
$$

namely the identity. By Theorem 2 we can conclude it is the only one, since for $R_{d}=\emptyset$ and $R_{s}$ consisting of the above four rules, the resulting TRS $\operatorname{Obs}\left(R_{s}\right)$ is terminating as can be proved by AProVE [4] or TTT2 [7]. Both [3] and [11] fail to prove that the identity is the only stream function satisfying the equation for $f$. By essentially choosing $\operatorname{Obs}\left(R_{s}\right)$ as the input and adding information about special contexts, the tool Circ [8] is able to prove that $f$ is the identity.

\section{$6 \quad$ Fixpoints}

Several streams are defined as fixpoints of stream functions, like the Fibonacci stream as given in the introduction. In our format it can be presented as the stream specification $R_{s}$ consisting of the rules

$$
\begin{aligned}
\text { Fib } & =f(\text { Fib }) & & g(0, \sigma)=0: 1: f(\sigma) \\
f(x: \sigma) & =g(x, \sigma) & & g(1, \sigma)=0: f(\sigma) .
\end{aligned}
$$

The TRS $\operatorname{Obs}\left(R_{s}\right)$ is not terminating since it allows the reduction

$$
\operatorname{head}(\text { Fib }) \rightarrow \operatorname{head}(f(\text { Fib })) \rightarrow \operatorname{head}(g(\text { head(Fib }) \text {, tail(Fib }))) .
$$

However, now we will polish $R_{s}$ to $R_{s}^{\prime}$ such that $\operatorname{Obs}\left(R_{s}^{\prime}\right)$ is terminating, by which well-definedness of both $R_{s}^{\prime}$ and $R_{s}$ can be concluded. This shows incompleteness of Theorem 1: the stream specification $R_{s}$ admits a unique model but $\operatorname{Obs}\left(R_{s}\right)$ is not terminating. 
Assume some model satisfies $R_{s}$; for simplicity we identify ground terms with their interpretations in the model. Then Fib $=f(\mathrm{Fib})=g(\cdots)=0: c$ for some stream $c$. Using this equality Fib $=0: c$ we obtain

$$
0: c=\mathrm{Fib}=f(\mathrm{Fib})=f(0: c)=0: 1: f(c),
$$

so $c=1: f(c)$. So the model also satisfies $R_{s}^{\prime}$ which is obtained from $R_{s}$ by replacing the first rule Fib $=f(\mathrm{Fib})$ by the two rules Fib $=0: c$ and $c=1: f(c)$. However, $R_{s}^{\prime}$ again satisfies our format and $\operatorname{Obs}\left(R_{s}^{\prime}\right)$ is terminating as can be proved by AProVE [4] or TTT2 [7]. So by Theorem $1 R_{s}^{\prime}$ admits a unique model, which is by construction the only model for $R_{s}$ too.

This technique of modifying the stream specification is generally applicable. If our technique fails for proving well-definedness of a stream specification, we can analyze the specified streams by applying the rules and deriving new equalities from which a modified stream specification can be composed. If our technique succeeds in proving well-definedness of the modified specification, conclusions can be drawn about the original one.

In general, stream functions may have zero, one or several fixpoints. For instance, the boolean stream function $f$ defined by

$$
f(0: \sigma)=0: 1: f(\sigma), \quad f(1: \sigma)=1: 0: f(\sigma),
$$

has two fixpoints: the Thue Morse stream morse from Example 1 and its inverse. Proving that there are exactly two can be done as follows. Assume $m$ is a fixpoint starting with 0 , so $m=0: c$. Then $0: c=m=f(m)=f(0: c)=0: 1: f(c)$, so $c=1: f(c)$. By adding the rules $m=0: c$ and $c=1: f(c)$ we have a stream specification $R_{s}$ for which termination of $\operatorname{Obs}\left(R_{s}\right)$ can be proved. So there is exactly one fixpoint of $f$ starting with 0 , and by symmetry there is exactly one fixpoint of $f$ starting with 1 .

\section{Conclusions}

We presented a technique by which well-definedness of stream specifications like

$$
\begin{aligned}
f(0: \sigma) & =1: f(\sigma) \\
f(1: \sigma) & =0: f(f(\sigma) \\
c & =1: c
\end{aligned}
$$

can be proved fully automatically, where a tool like Circ [8] fails, and the productivity tool [3] fails to prove productivity of $f(c)$. The main idea is to prove well-definedness by proving termination of a transformed system $\operatorname{Obs}\left(R_{s}\right)$, in this way exploiting the power of present termination provers.

We observed that productivity of the stream specification can not be concluded from termination of $\operatorname{Obs}\left(R_{s}\right)$; we leave as a challenge to find syntactic criteria on the stream specification by which this can be concluded.

Acknowledgments. We want to thank Venanzio Capretta, Joerg Endrullis, Herman Geuvers, Jan Willem Klop, Dorel Lucanu, Matthias Raffelsieper, Grigore Rosu and Alexandra Silva for fruitful discussions on this exciting topic, and the anonymous referees for fruitful suggestions. 


\section{References}

1. J.-P. Allouche and J. Shallit. Automatic Sequences: Theory, Applications, Generalizations. Cambridge University Press, 2003.

2. T. Arts and J. Giesl. Termination of term rewriting using dependency pairs. Theoretical Computer Science, 236:133-178, 2000.

3. J. Endrullis, C. Grabmayer, and D. Hendriks. Data-oblivious stream productivity. In Proceedings of the 11th International Conference on Logic for Programming, Artificial Intelligence, and Reasoning (LPAR'08), volume 5330 of Lecture Notes in Computer Science, pages 79-96. Springer, 2008. webinterface tool: http://fspc282.few.vu.nl/productivity/.

4. J. Giesl et al. Automated program verification environment (AProVE). Available at http://aprove.informatik.rwth-aachen.de/.

5. J. Giesl, R. Thiemann, and P. Schneider-Kamp. The dependency pair framework: Combining techniques for automated termination proofs. In Franz Baader and Andrei Voronkov, editors, Proceedings of the 11th International Conference on Logic for Programming, Artificial Intelligence, and Reasoning (LPAR'04), volume 3452 of Lecture Notes in Artificial Intelligence, pages 301-331, Montevideo, Uruguay, 2005. Springer.

6. J. Goguen, K. Lin, and G. Rosu. Circular coinductive rewriting. In Proceedings, 15th International Conference on Automated Software Engineering (ASE'00). Institute of Electrical and Electronics Engineers Computer Society, 2000. Grenoble, France, 11-15 September 2000, webinteface tool CIRC: http://fsl.cs.uiuc.edu/index.php/Special:CircOnline.

7. M. Korp, C. Sternagel, H. Zankl, and Aart Middeldorp. Tyrolean termination tool 2. In R. Treinen, editor, Proceedings of the 20th Conference on Rewriting Techniques and Applications (RTA), Lecture Notes in Computer Science. Springer, 2009. Tool available at http://colo6-c703.uibk.ac.at/ttt2/.

8. D. Lucanu and G. Rosu. CIRC: A circular coinductive prover. In CALCO'07, volume 4624 of Lecture Notes in Computer Science, pages 372 - 378, 2007.

9. C. Marche and H. Zantema. The termination competition. In F. Baader, editor, Proceedings of the 18th Conference on Rewriting Techniques and Applications (RTA), volume 4533 of Lecture Notes in Computer Science, pages 303-313. Springer, 2007.

10. Grigore Roşu. Equality of streams is a $\Pi_{2}^{0}$-complete problem. In Proceedings of the 11th ACM SIGPLAN International Conference on Functional Programming (ICFP'06). ACM, 2006.

11. J.J.M.M. Rutten. A coinductive calculus of streams. Mathematical Structures in Computer Science, 15:93-147, 2005.

12. J. G. Simonsen. The $\Pi_{2}^{0}$-completeness of most of the properties of rewriting systems you care about (and productivity). In R. Treinen, editor, Proceedings of the 20th Conference on Rewriting Techniques and Applications (RTA), Lecture Notes in Computer Science. Springer, 2009. 